\title{
CONTROL INTERNO EN LA EJECUCIÓN DE GASTOS DE INVERSIÓN DE LA MUNICIPALIDAD DE INDEPENDENCIA - ANCASH
}

\author{
INTERNAL CONTROL IN THE INVESTMENT EXPENSES EXECUTION OF \\ THE MUNICIPALITY OF INDEPENDENCE - ANCASH \\ Yaneth Zenaida Montes EspinozA* \\ Unidad de Posgrado de la Facultad de Ciencias Contables \\ Universidad Nacional Mayor de San Marcos-UNMSM / Lima-Perú \\ [Recepción: Febrero de 2014 / Conformidad: Febrero de 2014]
}

\section{RESUMEN}

La aplicación del control interno en la Municipalidad Distrital de Independencia (MDI) en Ancash no contribuye al alcance de sus objetivos y metas establecidos en la ejecución de gastos destinados para proyectos de inversión; durante el periodo 2007 - 2011, la Entidad no culminó la ejecución de proyectos de inversión programados en el Presupuesto Institucional de Apertura (PIA) y el Presupuesto Operativo Institucional (POI), a pesar de que contó con presupuesto disponible, generando reclamos y malestar en la población, en algunos casos en zonas emergentes. El presente estudio, ha demostrado que los factores tales como: el personal no idóneo, el incumplimiento de normas y políticas, el deficiente control de sus recursos, incluyendo la actitud de la dirección de la entidad, afectaron negativamente la aplicación de las políticas de control interno en la ejecución de gastos de proyectos de inversión.

\section{Palabras clave:}

Proyectos de inversión, control interno, gasto, presupuesto, ejecución.

\begin{abstract}
The implementation of internal control in the District Municipality of Independence (DMI) in Ancash does not contribute to achieving its objectives and targets established in the expenses execution for investment projects; during the 2007 - 2011 period, the Entity did not complete the execution of investment projects scheduled in the Opening Institutional Budget (PIA) and the Institutional Operating Budget (POI), although it had available budget, generating complaints and discomfort in the population, in some cases in emerging areas. This study has shown that factors such as non-qualified personnel, failure of standards and policies, poor control of their resources-including the attitude of the entity's management - affected negatively on the implementation of control policies in the expenses execution of investment projects.
\end{abstract}

\section{Keywords:}

Investment projects; internal control; expenses; budget; execution.

* Maestrista en Auditoría con Mención en Auditoría en la Gestión y Control Gubernamental - UNMSM. Email: yanethme29@hotmail.com 


\section{INTRODUCCIÓN}

En el marco de la Constitución Política de nuestro país, específicamente los artículos $\mathrm{N}^{\circ} \mathrm{s} 77^{\circ}, 81^{\circ} \mathrm{y}$ $82^{\circ}$, la administración económica y financiera de los recursos destinados para los gobiernos regionales, se rigen por los presupuestos anuales aprobados por el congreso; y para garantizar una eficiente ejecución de los recursos destinados, el órgano superior del Sistema Nacional de Control, que es la Contraloría General de la República, supervisa la legalidad de las operaciones de la deuda pública y de los actos de las instituciones sujetas a control.

En ese sentido, el presente trabajo de investigación titulado "Control Interno y su incidencia en la ejecución de gastos de proyectos de inversión de la Municipalidad Distrital de Independencia en Ancash", tiene como objetivo determinar en qué medida la aplicación del control interno constituye un instrumento eficaz para el mejoramiento en la ejecución de gastos destinados para proyectos de inversión.

Los controles internos se implantan con el fin de detectar, en el plazo deseado, cualquier desviación respecto a los objetivos de rentabilidad establecidos por las instituciones y de limitar ciertas desviaciones. Asimismo, los controles internos fomentan la eficiencia, eficacia y economía de los recursos, reducen el riesgo de pérdida de valor de los activos y ayudan a garantizar la fiabilidad de los estados financieros y el cumplimiento de las normas legales vigentes ${ }^{1}$.

Ochoa y Charris (2003), en su investigación "Propuesta de un Modelo de Control Fiscal para el Estado Colombiano: El Sistema de Control Fiscal Nacional", afirma que hay problemas serios en el logro de los objetivos que señalan la constitución, las leyes y los decretos para el ejercicio del control fiscal. Existe una baja calidad en el control fiscal, en especial en los departamentos y en los municipios, lo cual no garantiza la conservación de los activos del Estado ni transparencia en las transacciones.

Tomando en consideración la importancia que tiene el control interno en la verificación de las actividades que se realizan a nivel organizacional; el presente estudio contribuirá para que se corrijan las deficiencias encontradas en la aplicación de políticas de control interno en la ejecución de gastos destinados para proyectos de inversión, constituyéndose en un aporte a la solución de los problemas identificados en la entidad de estudio, y a la sociedad peruana en general.

\section{PROBLEMA PRINCIPAL}

¿En qué medida la aplicación del control interno constituye un instrumento eficaz para el mejoramiento en la ejecución de gastos en proyectos de inversión en la Municipalidad Distrital de Independencia en Ancash?

\section{PROBLEMAS ESPECÍFICOS}

a) ¿Cuáles son los factores que afectan la aplicación de las políticas de control interno en la ejecución de gastos de proyectos de inversión?

b) ¿El control interno permite alcanzar los objetivos y metas establecidos en la ejecución de gastos de proyectos de inversión?

c) ¿La sobrevaloración en la ejecución de obras, se debe a que los presupuestos programados y aprobados en el PIA son cargados indebidamente a gastos administrativos?

\section{BASES TEÓRICAS}

\section{Control Interno}

El control interno se define como un proceso efectuado por el consejo de administración, la dirección y el resto del personal de una entidad, diseñado con el objeto de proporcionar un grado de seguridad razonable en cuanto a la consecución de objetivos dentro de las siguientes categoría:

- Eficacia y eficiencia de las operaciones.

- Confiabilidad de la información financiera.

- Cumplimiento de las leyes y normas que sean aplicables.

La primera categoría, se dirige a los objetivos empresariales básicos de una entidad, incluyendo los objetivos de rendimiento y la rentabilidad y la

1 Ley No 28716, Ley de Control Interno de las Entidades del Estado, Art. $4^{\circ}$. Separata Especial de Normas Legales del Diario Oficial "El Peruano", Lima - Perú, 18 de abril de 2006, Página 316872.

82/ QuipuramaVoc | Vol. 23(43) 2015 
salvaguarda de los recursos. La segunda, está relacionada con la elaboración y publicación de estados financieros confiables, incluyendo estados financieros interinos y abreviados, así como la información financiera extraída de dichos estados, por ejemplo, los comunicados sobre resultados que sean publicados. La tercera, concierne al cumplimiento de aquellas leyes y normas a las que está sujeta la entidad. Estas distintas pero, en parte, coincidentes categorías tratan diferentes necesidades y permiten un enfoque dirigido hacia la satisfacción de las necesidades individuales.

Los sistemas de control funcionan en tres niveles distintos de eficacia. El control interno se puede considerar eficaz en cada una de las tres categorías, respectivamente, si el consejo de administración y la dirección tienen la seguridad razonable de que:

- Disponen la información adecuada sobre hasta qué punto se están logrando los objetivos operacionales de la entidad.

- Se preparan de forma confiable los estados financieros públicos.

- Se cumplen las leyes y normas aplicables.

Si bien el control interno es un proceso, su eficacia es el estado o la situación del proceso en un momento dado.

Según la Ley No 287785, Ley Orgánica del Sistema Nacional de Control y de la Contraloría General de la República, articulo13, el Sistema Nacional de Control está conformado por los siguientes órganos de control:

a) La Contraloría General, como ente técnico rector.

b) Todas las unidades orgánicas responsables de la función de control gubernamental de las entidades.

c) Las sociedades de auditoría externa independientes, cuando son designadas por la Contraloría General.

\section{Ejecución de Proyectos de Inversión}

Para el presente estudio, un proyecto de inversión debe cumplir con las características de ser sostenible en el tiempo que implique ampliar, mejorar y modernizar la capacidad productora de bienes o prestadora de servicios efectuados por la Municipalidad Distrital de Independencia.

\section{METODOLOGÍA}

Es una investigación descriptiva y explicativa, porque nos permite conocer las características más relevantes del problema, detallando deficiencias existentes por la falta de un sistema de control interno, con la cual se pretende dar recomendaciones con el fin de mejorar la toma de decisiones en la gestión de los recursos destinados para proyectos de inversión.

\section{RESULTADOS Y DISCUSIÓN}

Se realizó la encuesta a cada uno de los trabajadores de las áreas involucrados con el control interno y la ejecución de proyectos de inversión de la Municipalidad Distrital de Independencia, obteniéndose para cada Problema Especifico (PE1, PE2 y PE3), el resultado siguiente:

Factores que afectan la aplicación de las políticas de control interno en la ejecución de gastos de proyectos de inversión (PE1)

- El personal con que cuenta la Municipalidad de Independencia en las áreas como: Planificación y Presupuestos, Desarrollo Urbano, y Oficina de Proyectos de Inversión, no son los más idóneos, debido a que se contrató personal sin previa evaluación, y calificación curricular y de conocimientos, tal como se verifica en el resultado del Gráfico $\mathrm{N}^{\circ} 01$, en la que el $58 \%$ del personal encuestado afirmaron la situación expuesta.

- La municipalidad no propicia la participación permanente del personal a cursos de capacitación $\mathrm{y}$ actualización en normas de control, proyectos de inversión y materia presupuestal, por lo que sus niveles de conocimiento son escasos, y no permite que el personal encargado de la ejecución de gastos en proyectos de inversión y materia presupuestaria cumplan adecuadamente con sus funciones. $\mathrm{Al}$ respecto, el 79\% del personal encuestado manifestaron que no asisten o asisten muy pocas veces a los cursos de capacitación y actualización en normas de control, proyectos de inversión y materia presupuestal. 
Gráfico No 01: ¿Participa en curso o evento de capacitación referidos a normas de control y proyectos de inversión?

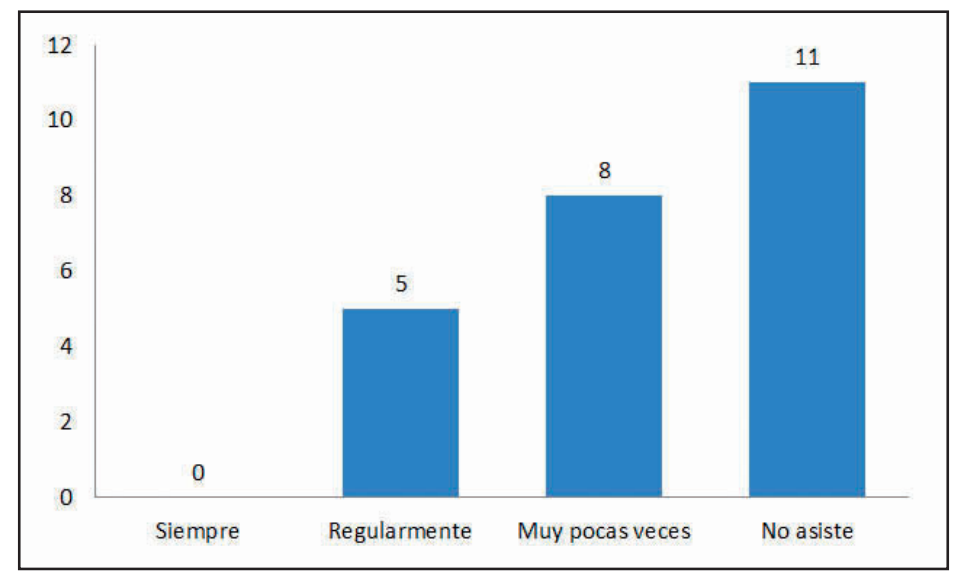

Fuente: Encuesta. Elaboración propia.

- Las normas y disposiciones internas con que cuenta la Entidad no se encuentran debidamente actualizadas acorde con las normas generales vigentes. Asimismo, no se encuentran debidamente publicadas y divulgadas al alcance y conocimiento de todo el personal; hecho que no permiten que el personal encargado de la ejecución presupuestaria, administración de gastos, así como la ejecución de obras, no cumpla sus funciones con eficacia, eficiencia, transparencia y economía (Ver Gráfico No 02).

Gráfico No 02: ¿Las normas y disposiciones internas con que cuenta la entidad se encuentran debidamente publicadas y divulgadas al alcance y conocimiento de todo el personal?

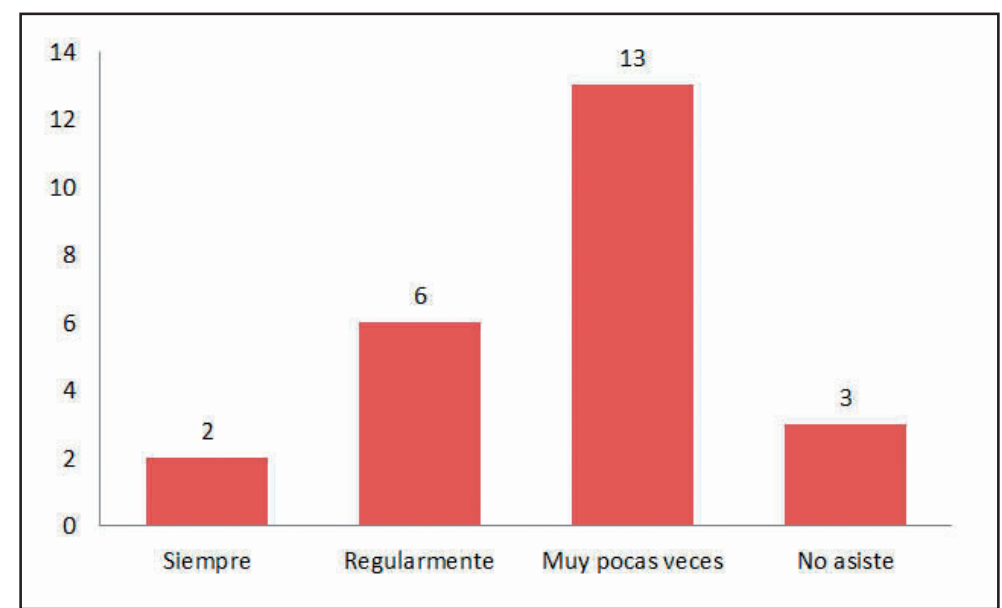

Fuente: Encuesta. Elaboración propia.

\section{Determinar si el control interno permite} alcanzar los objetivos y metas establecidos en la ejecución de gastos de proyectos de inversión (PE2)

- Las fuentes y montos de los recursos asignados anualmente para financiar los planes, programas y proyectos de la municipalidad, pocas veces cumplen con las metas y los objetivos previstos, debido a que el Presupuesto de Organización Institucional (POI) y Presupuesto Institucional de Apertura (PIA) con el que cuenta la municipalidad no son ejecutados en su total, tal como verifica en el resultado del Gráfico $\mathrm{N}^{\circ} 03$. 
Gráfico No 03: ¿Las fuentes y los montos de los recursos asignados anualmente para financiar los proyectos de la Municipalidad, cumplen con los objetivos previstos?

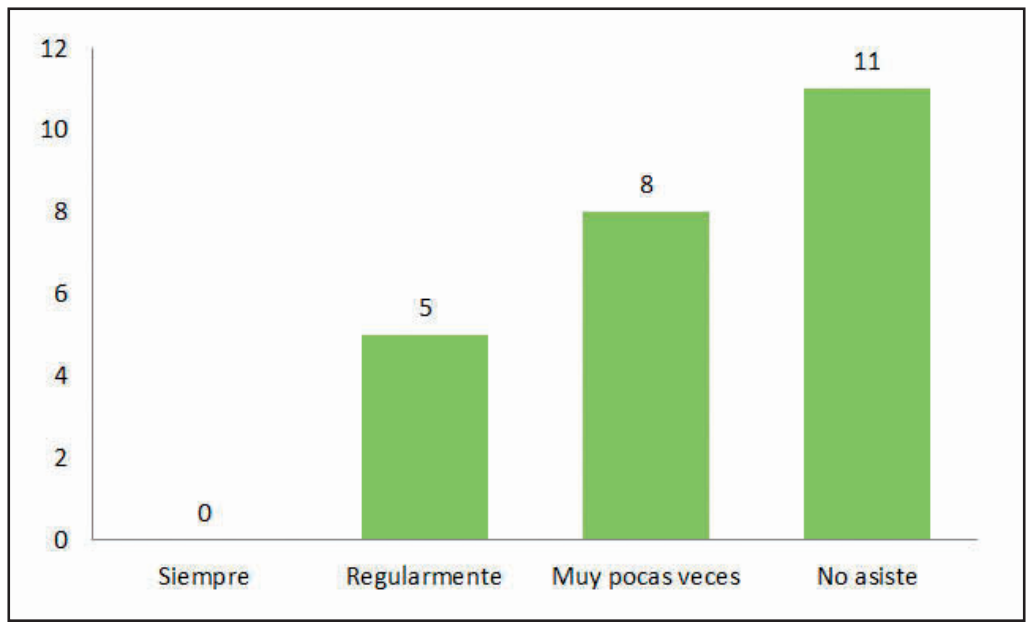

Fuente: Encuesta. Elaboración propia.

- Las recomendaciones emitidas por los órganos del Sistema Nacional de Control, como es la Órgano de Control Institucional, u otros entes de fiscalización que correspondan, no han sido implementadas oportunamente, lo que no le permitió identificar, analizar y administrar ade- cuadamente los factores o eventos que puedan afectar adversamente el cumplimiento de los fines, metas, objetivos, actividades y operaciones de la municipalidad. Al respecto, el $63 \%$ del personal encuestado afirmaron dicha situación (Ver Gráfico $\left.\mathrm{N}^{\circ} 04\right)$.

Gráfico No 04: ¿Las recomendaciones emitidas por la propia entidad (informe autoevaluación), los órganos del Sistema Nacional de Control y otros entes de fiscalización que correspondan son implementadas oportunamente?

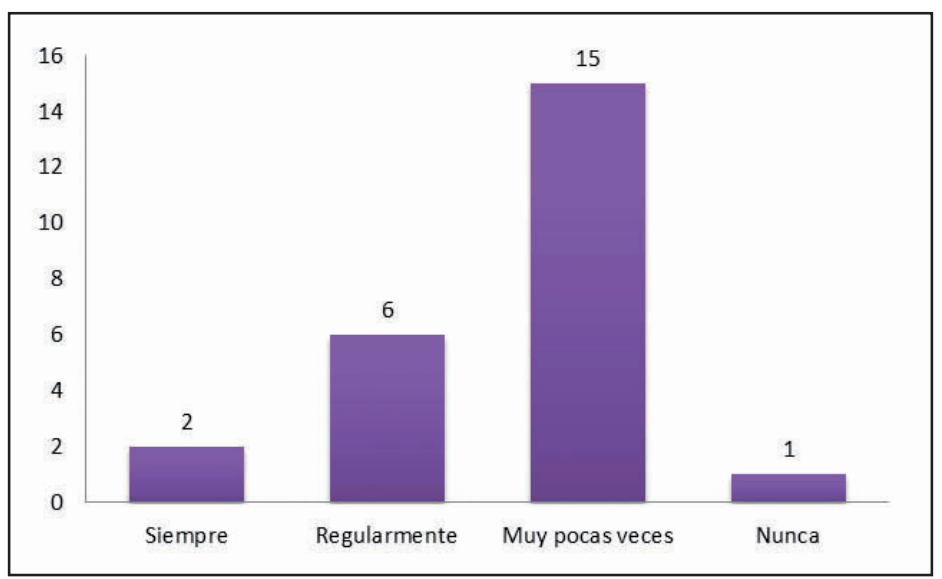

Fuente: Encuesta. Elaboración propia. 
La sobrevaloración en la ejecución de obras, se debe a que los gastos programados y aprobados en el PIM son cargados indebidamente a otros proyectos o actividades (PE3)

- La Entidad no hace seguimientos y controles continuos a los procedimientos de ejecución de gastos de los proyectos de inversión, así como, tampoco cuenta específicamente con una directiva interna que regule procedimientos para la elaboración y ejecución presupuestaria, liquidaciones financieras, y ejecución de gatos en los Proyectos de Inversión; lo que conllevó a que se realizaran
Anulaciones y/o Modificaciones presupuestarias sin autorización y aprobación de la Gerencia de Planificación y Presupuestaria, incumpliendo la normatividad vigente en materia presupuestaria, y que además, el importe presupuestado de muchos proyectos y obras sean modificados para ser destinados para otros proyectos, o en el peor de los casos, sean destinados en gastos administrativos o gastos corrientes; ocasionando que la municipalidad tenga una sobrevaloración en muchas obras ejecutadas durante el periodo 2007- 2011. Situación que fue confirmado por más del $50 \%$ del personal encuestado (Ver Gráficos Nos 05 y 06).

Gráfico No 05: ¿ Se hacen seguimientos y controles continuos a los procedimientos de ejecución de gastos de los proyectos de inversión

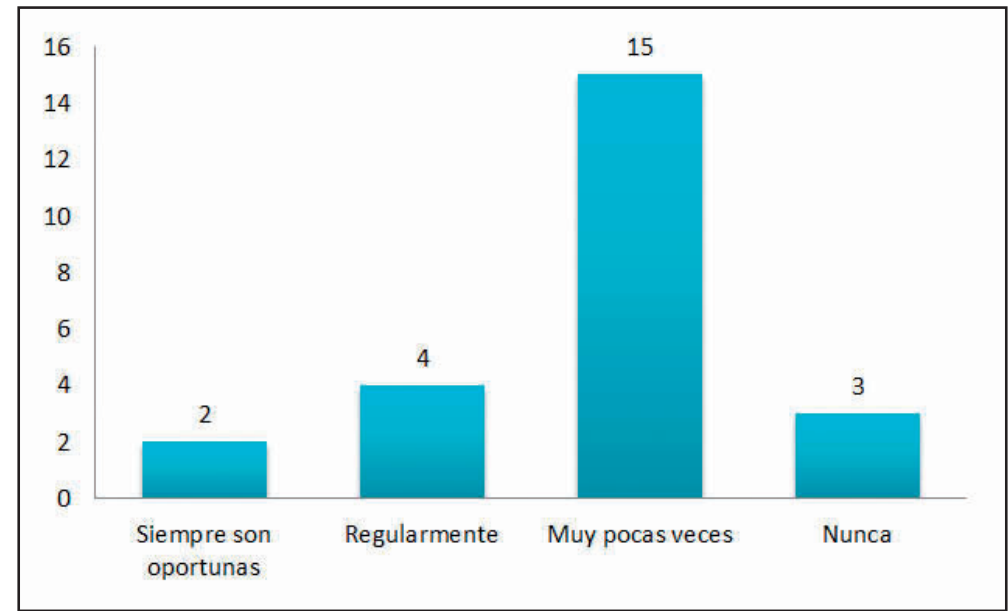

Fuente: Encuesta. Elaboración propia.

Gráfico No 06: ¿Todos los gastos destinados a proyectos de inversión son ejecutados en Actividades de Inversión?

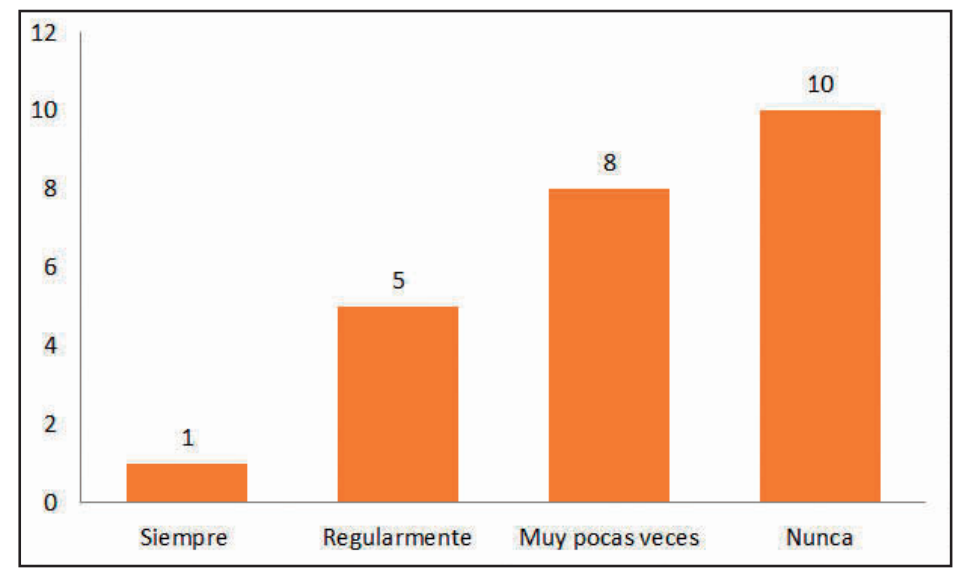

Fuente: Encuesta. Elaboración propia. 


\section{CONCLUSIONES}

1. La Municipalidad Distrital de Independencia en Ancash presentó un deficiente sistema de control interno respecto a la ejecución de los proyectos de inversión durante el periodo 2007 - 201 1; debido a que factores, tales como: el incumplimiento de política y normas establecidas, la falta de publicación y actualización de las directivas y normas internas existentes, la falta de control de sus recursos destinados para proyectos de inversión, y la contratación directa del personal en su mayoría sin realizar convocatorias públicas; afectaron negativamente la aplicación de las políticas de control interno en la ejecución de gastos de proyectos de inversión.

2. El débil y bajo nivel de control interno con que cuenta la Municipalidad Distrital de Independencia en Ancash, no permitió el alcance de los objetivos y metas establecidos en la ejecución de gastos destinados para proyectos de inversión durante el periodo 2007 - 2011. Como consecuencia, la Entidad no culminó la ejecución de proyectos de inversión programados en el Presupuesto Institucional de Apertura (PIA) y el Presupuesto Operativo Institucional (POI), no obstante que se contó con presupuesto disponible para todos los proyectos programados. Esta situación ha generado que la ciudadanía en su conjunto no esté conforme con la cantidad y la calidad de proyectos ejecutados; en algunos casos en zonas emergentes, originando una serie de malestares y reclamos por parte de la población.

3. La sobrevaloración en la ejecución de obras de la Municipalidad Distrital de Independencia en Ancash, se debe a que los gastos programados y aprobados en el Presupuesto Institucional de Apertura (PIA) son modificados mediante habilitaciones y anulaciones para ser cargados indebidamente a gastos administrativos; asimismo, se debe al otorgamiento de adicionales de obra de manera excesiva, y la falta de procedimientos internos que facilite el eficiente desarrollo de sus actividades u operaciones en la ejecución de obras (Objetivo 3).

\section{REFERENCIAS BIBLIOGRÁFICAS}

1. CASTILLO, J. (2006) Centro de Estudios Gubernamentales. Administración Pública 2006. Tomo I. Edición: Lima - Perú 2006.

2. Committee of Sponsoring Organizations (COSO) of the Treadway Commission - CONTROL INTERNO - Estructura Conceptual Integrada.

3. Constitución Política del Perú 1993. Lima. Editora Perú.

4. Contraloría General de la República (2001) Ley $\mathrm{N}^{\circ} 27785$, Ley Orgánica del Sistema Nacional de Control y de la Contraloría de la Republica. Lima. Editora Perú.

5. Contraloría General de la República (2004) Ley $\mathrm{N}^{\circ}$ 2871, Ley de Control Interno de las Entidades del Estado. Lima: Editora Perú.

6. Contraloría General de la República (1992) Manual de Auditoria Gubernamental (MAGU). Lima. Editora Perú.

7. Contraloría General de La República (1992) Normas de Auditoría Gubernamental (NAGUS). Lima. Editora Perú.

8. Contraloría General de la República (2001) Directiva $\mathrm{N}^{\circ}$ 013-2001-CG/B340 - “Disposiciones para la Auditoría a la Información Financiera y al Examen Especial a la Información Presupuestaria preparada para la Cuenta General de la República por las Entidades del Sector Publico". Lima. Editora Perú.

9. Ministerio de Economía y Finanzas (2003) Ley $\mathrm{N}^{\circ} 28112$, Ley Marco de la Administración Financiera del Sector Publico". Lima. Editora Perú.

10.Ministerio de Economía y Finanzas. (2010) Ley $\mathrm{N}^{\circ} 29465$, Ley de presupuesto del sector público para el año fiscal 2010. Lima. Editora Perú.

11.Ministerio de Economía y Finanzas (2004) Ley $\mathrm{N}^{\circ}$ 28411, Ley General del Sistema Nacional de Presupuesto. Lima. Editora Perú. 
12. Municipalidad Distrital de Independencia. (2009) Presupuesto Institucional Modificado (PIM).

13. OCHOA, D. CHARRIS, S. (Octubre / diciembre, 2003). Propuesta de un modelo de control Fiscal para el Estado Colombiano: El sistema de control Fiscal Nacional. Revista Estudios Gerenciales, (89), pp. 13-42.
14. R. K. Mautz y HUSSEIN A. Sharaf. (1971) American Accountig Association. La filosofía de la Auditoria. Ediciones Contables y Administrativas. Primera Edición. 